Note: Positive action is an alteration of course and/or speed which causes an anti-clockwise rotation of the sight-line between the two vessels if the other vessels stands-on. Negative action causes the sight-line to rotate in a clockwise direction.

Resumption of course. After turning to starboard for a vessel on the starboard side keep the vessel to port when resuming course.

Escape action. A vessel approaching from the port side and rear sector can normally be expected to take early avoiding action. The suggested turns are recommended for use when such a vessel fails to keep out of the way. As an alteration to put the bearing astern may not complement subsequent action by the other vessel, it is recommended that further turns be made to keep the vessel astern until she is well clear.

SPEED ChANGes IN Restricted visibility. Reductions of speed. A vessel can reduce speed or stop at any time and such action is recommended when the compass bearing of a vessel on the port bow is gradually changing in a clockwise direction (increasing). A reduction of speed should be made as an alternative to, and not in conjunction with, the suggested turn to starboard for avoiding a vessel either on the port bow or ahead. Normal speed should be resumed if it becomes apparent that a vessel on the port side has either subsequently turned to starboard in order to pass astern, or stopped.

Increases of speed. It will sometimes be advantageous to increase speed if this is possible within the limitations of the requirement to proceed at a safe speed. An increase of speed may be appropriate when the vessel to be avoided is astern, or on the port quarter, or near the port beam, either initially or after taking the alter course action indicated in the diagram.

Limitations. The presence of other vessels and/or lack of sea room may impose limitations on the manœurres which can be made, but it should be kept in mind that small changes of course and/or speed are unlikely to be detected by radar.

Caution. It is essential to ensure that any action taken is having the desired effect. If not, the recommended turns can normally be applied successively for newly developed collision situations with the same vessel.

\title{
Historical Hyperbolae
}

\section{Claud Powell}

Ar a first glance, one of the two accompanying diagrams might be taken to represent a three-station Decca or Loran chain, while the other offers familiar instruction on how to interpolate between the lattice lines and plot the fix. In fact, these are illustrations in a German Army handbook entitled An Introduction to Sound Ranging and the Work of the Sound Ranging Section, published in July 19 I 8.

$\mathrm{A}, \mathrm{B}$ and $\mathrm{C}$ are interconnected receiving stations (microphones) on baselines of about $\mathrm{I} \mathrm{km}$. and their object is to find the position of a hostile gun by comparing the times at which the sound of its report arrives at the three known positions. Assuming a constant propagation speed, comparing the three observations yields a pair of hyperbolic position lines whose intersection gives the gun's position. As in Loran the lattice lines are numbered directly in units of time difference 

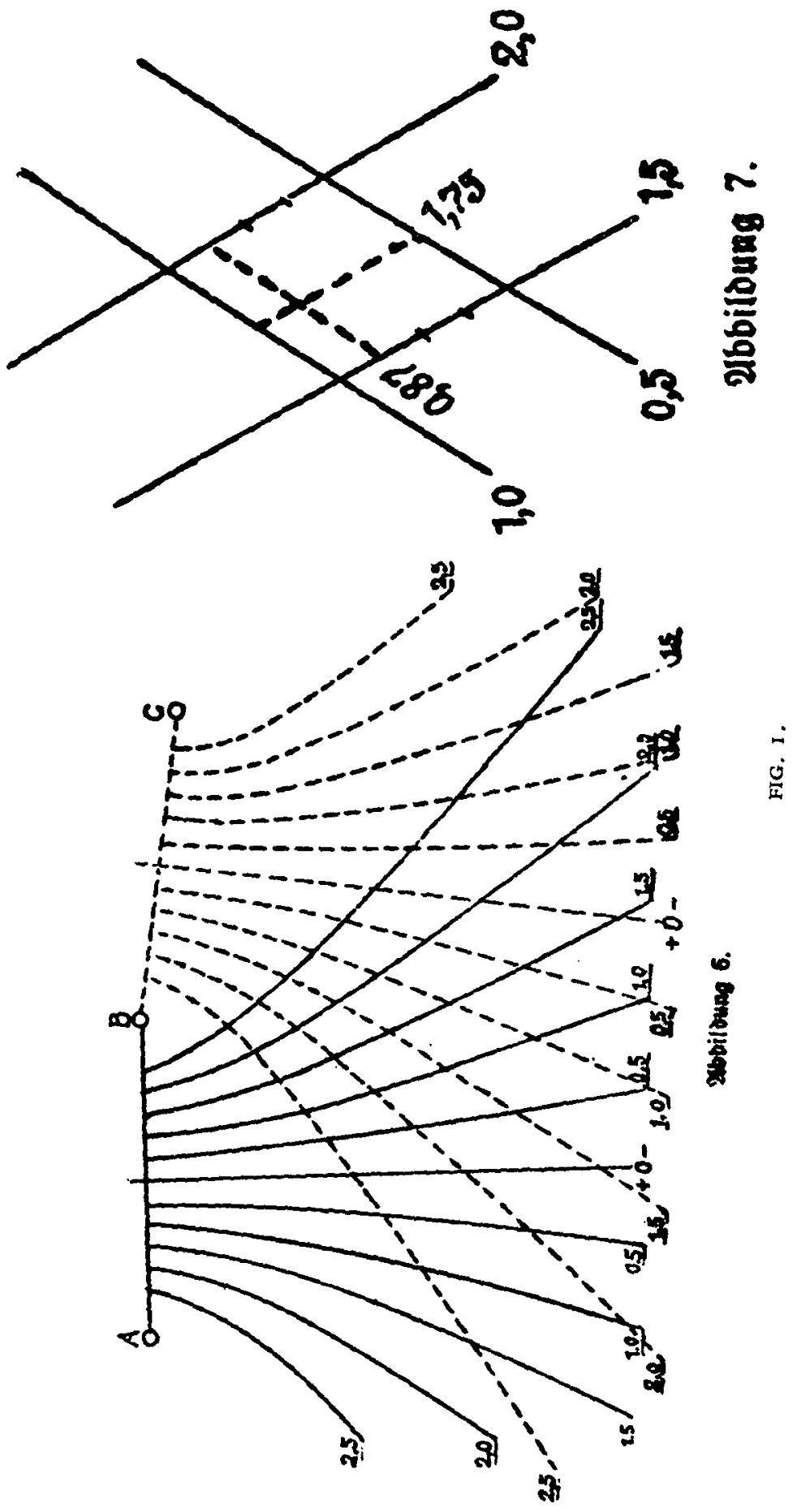
(seconds rather than microseconds), but there is no 'coding delay' and logically enough the centre line is marked zero. Thus if the sound reached A and B simultaneously the gun would be somewhere on the centre line of that pattern and if it reached $B$ exactly $1 \frac{1}{2}$ seconds before $C$, the dotted $\times .5$ second line in the 'plus' half of the $\mathrm{BC}$ pattern would be the other position line. The fix is plotted by interpolating to $\mathrm{I} / \mathrm{I}$ oo second as illustrated.

Sound ranging was started, on both sides, in the first World War. The Allies used a different microphone layout and resolved the time differences without recourse to a pre-drawn family of constant-time-difference lines. The Germans tried various techniques including the one shown here and are said to have used coloured lattice overprints, of which I hope to find an example. Sound ranging is still used, but the lattice method of plotting was short-lived; in a wider context, however, it was strikingly prophetic.

It was through the kindness of the late Sir Lawrence Bragg, himself one of the pioneers of sound ranging, in allowing me access to his papers, that I learned of the German work. I am grateful to Oberstleutnant A. Pilgram of the School of Artillery at Idar-Oberstein, West Germany, for supplying a photostat copy of the 1918 handbook.

\section{Distance by Vertical Angle}

\section{Charles H. Cotter}

THE note by V. L. Bosazza (on page 112 ) raises a number of interesting points. First it must be noted that there is no indication in Ryder's book 1 that the horizon method, viz. 'To observe from the cross-trees or other convenient place the angle subtended between the horizon and the enemy's waterline', was used before it was introduced by Ryder in 1845 . Ryder remarked that in not a single man-of-war which he visited before the time of the publication of his book did he find any method in use that would denote satisfactorily the range of a ship at sea; and it was to meet this deficiency that he suggested his horizon method.

Ryder described the methods in general use before the introduction of the horizon method, and in so doing drew attention to their defects from the gunner's point of view. Any of these methods may well have had their applications in maritime surveying during the nineteenth century; but of course surveying was not the immediate concern of Ryder.

The method devised by Sir Howard Douglas, in which the vertical angle between an enemy's masthead and waterline was measured with a sextant, was useless unless the height of the enemy's mast was known. In connection with Douglas's method, Ryder suggested the use of a micrometer telescope and mentioned that the hydrographic department of the Admiralty then supplied Rochon's micrometer telescopes to surveying vessels. Moreover Ryder informs us in a footnote that:

'Mr. Blakey, a master in the Royal Navy, has made a telescope without glasses, but with fine wires, by means of which the distance from ships of known height can be readily ascertained.' 